\title{
Disturbance Attenuation in Linear Systems Revisited
}

\author{
Jiqiang Wang ${ }^{\text {a }}$, Hong Yue ${ }^{\mathrm{b}}$, Georgi Dimirovski ${ }^{\mathrm{c}}$ \\ $a$ Jiangsu Province Key Laboratory of Aerospace Power Systems, College of Energy and \\ Power Engineering, Nanjing University of Aeronautics and Astronautics, 29 Yudao Street, \\ Nanjing 210016, China \\ E-mail: jiqiang.wang@nuaa.edu.cn \\ $b$ Department of Electronic \& Electrical Engineering, University of Strathclyde, Glasgow, \\ UK \\ Email: hong.yue@strath.ac.uk \\ $c$ Dogus University of Istanbul, Istanbul 34722, Turkey \\ Email: gdimirovski@ dogus.edu.tr \\ * Corresponding author: Jiqiang Wang, Room 603, Building 11, 33 Jiangjun Dadao, \\ Jiangning District, Nanjing 211100, China \\ E-mail address: jiqiang.wang@ nuaa.edu.cn \\ Or: jiqiang_wang@hotmail.com
}

Tel: +861524021 1717. Fax: +862584893666. 


\title{
Disturbance Attenuation in Linear Systems Revisited
}

\author{
Jiqiang Wang ${ }^{\mathrm{a}}{ }^{*}$, Hong Yue ${ }^{\mathrm{b}}$, Georgi Dimirovski ${ }^{\mathrm{c}}$ \\ a Jiangsu Province Key Laboratory of Aerospace Power Systems, College of Energy and Power Engineering, Nanjing \\ University of Aeronautics and Astronautics, 29 Yudao Street, Nanjing 210016, China \\ E-mail: jiqiang.wang@ nuaa.edu.cn \\ $b$ Department of Electronic \& Electrical Engineering, University of Strathclyde, Glasgow, UK \\ Email: hong.yue@strath.ac.uk \\ $c$ Dogus University of Istanbul, Istanbul 34722, Turkey \\ Email: gdimirovski@dogus.edu.tr
}

\begin{abstract}
A variety of methods have been proposed for attenuating or rejecting disturbances in linear systems. Most of the approaches, however, are targeting the performance either near resonance or across the whole frequency range. Weighting functions can be utilized to shape the frequency response function over target frequency band but they are usually of rule-of-thumb nature. A methodology is necessitated for designing controllers with performance specified at any discrete frequency or over any desired frequency band. The paper aims to develop such a methodology. Besides this, the proposed method can tell performance limitations and determine the problem of existence of optimal controllers, as well as providing a useful framework to improve the performance of an existing controller. A number of important results are obtained and these results are subsequently validated through a practical application to a rotor blade example.
\end{abstract}

Key words: disturbance attenuation, discrete frequency control, performance limitation \& improvement.

\section{Introduction}

Disturbance attenuation/rejection represents one of the most important problems in control system design. Conventional approach to disturbance attenuation is the classical sensitivity shaping that eventually cultivates into a unified regulator theory in a generalized plant setup [1-3]. In this setup, the disturbance attenuation concerns the problem of minimizing the gain (usually in $H_{2} / H \infty$ norm) from disturbance input to regulated output $[4,5]$. The $H_{2} / H \infty$ optimization methodology is powerful since it can also handle constraints by formulating into LMIs [6]. Indeed, within this framework, there exists numerous literature on tackling the disturbance attenuation problem (subject to uncertainty, saturation, delay etc), e.g. via state feedback, dynamic feedback, eigenstructure assignment, adaptive controls etc. In fact, this $H_{2} / H \propto$ optimization methodology to disturbance attenuation has been extended into nonlinear systems [7, 8], discrete event and multi-agent systems $[9,10]$, hybrid dynamical systems [11-13] and networked control systems [14-16] etc.

The above methodology, however, aims to achieve minimization of $H_{2} / H \propto$ norm over all frequencies. Typical filter design exists for shaping/weighting the frequency range of interest, e.g. the $H \infty$ optimization tries to "bring down" the peak magnitude of the frequency response function, but it is not transparent for the design

\footnotetext{
* Corresponding author. Tel: +86 1524021 1717; fax: +86 2584893666.
} 
methodology on how to shape the performance over a frequency band $\left[\begin{array}{ll}\omega_{1}, & \omega_{N}\end{array}\right]$, while allowing performance deterioration outside $\left[\omega_{1}, \omega_{N}\right]$.

While $H_{2} / H \infty$ optimization methodology to disturbance attenuation arises from control community, there are different approaches coming from noise and vibration control community. In this field, disturbance attenuation is mainly handled through internal model principle and adaptive disturbance estimation (see the review article [17]). The performance index is usually defined as of a minimum variance type (with weightings), which is essentially equivalent to minimizing a weighted mean square error [18-20]. Henceforth, the approaches are not targeting the performance defined over a frequency band. Even though the minimum variance type of square errors is defined at narrow band frequencies, simply synthesizing an optimal controller using optimal control methodology does not provide information on important problems such as the limit of performance at the desired frequency. In fact, the problem of determining limit of performance is an important issue that has unfortunately overlooked for many design approaches. The mixed sensitivity method takes care of this issue by minimizing $H_{2} / H \infty$ norm for both sensitivity $S$ and complementary sensitivity $T$ [21, 22]. But the choice of weighting functions for both $S$ and $T$ is of rule-of-thumb nature, aiming to maintain the ideal shapes for the norms of sensitivity functions (e.g. $S$ small in low frequencies and $T$ small over high frequencies etc). Important intuition on both solution existence and limit of performance at any discrete frequency $\omega_{0}$ or over a frequency band $\left[\omega_{1}, \omega_{N}\right]$ is thus lost.

Therefore, for disturbance attenuation, a methodology is necessitated that can possess the following features and capabilities simultaneously:

(1) Allow disturbance attenuation at any specific frequency or over any desired frequency band;

(2) Allow explicit determination of performance limitation for any frequency or over any desired frequency band;

(3) Allow performance improvement to current control.

In specific, feature (1) will provide a systematic sensitivity shaping method other than using weighting functions; feature (2) is even important, since prior to control design, a series of significant questions can be resolved, such as: 1) does a controller exists that suppresses disturbance in both $S$ and $T$, and by how much (in $\mathrm{dB}$ )? 2) Does a controller exists that will annihilate $S$ without enhancement of $T$ ? And similarly 3) Does a controller exists that will annihilate $T$ without enhancement of S? Also 4) Can a controller be found that will suppress $S$ whenever $T$ is attenuated, and vise versa?

Feature (3) will be extremely important for the situation where the disturbance is known to be in a specific frequency band (but not necessarily being able to be modeled or even measurable). Then the proposed method can be utilized to further enhance the controller performance over the desired frequency band, while accepting performance deterioration outside that frequency band (due to Bode's integral relationships or waterbed effect). In fact, no control design methodology is capable of exploiting performance limitation over a specific and explicit frequency band, but it can be demonstrated that the proposed method can always improve the current control design, be it $H_{2} / H \infty$, PID, or adaptive control etc over an explicitly defined frequency band $\left[\omega_{1}, \omega_{N}\right]$. In this sense, the proposed method is claimed to be UNIVERSAL to performance improvement at any discrete frequency or over any desired frequency band. The above features form the contribution of the current paper. 
They will be explained in the following sections: section 2 formulates the problem; section 3 and section 4 consider the discrete frequency control and broad band control respectively; the above theoretical results are validated through their application to a rotor blade structure in section 5, while a comparative study with optimal control design is carried out in section 6. Finally, section 7 concludes the paper.

\section{Problem Formulation}

To fix the discussion, the systems dynamics is described by the following frequency response functions:

$$
\left[\begin{array}{c}
y(j \omega) \\
z_{1}(j \omega) \\
z_{2}(j \omega) \\
\vdots \\
z_{n}(j \omega)
\end{array}\right]=\left[\begin{array}{cccc}
G_{00}(j \omega) & G_{01}(j \omega) & \cdots & G_{0 n}(j \omega) \\
G_{10}(j \omega) & G_{11}(j \omega) & \cdots & G_{1 n}(j \omega) \\
G_{20}(j \omega) & G_{21}(j \omega) & \cdots & G_{2 n}(j \omega) \\
\vdots & \vdots & \ddots & \vdots \\
G_{n 0}(j \omega) & G_{n 1}(j \omega) & \cdots & G_{n n}(j \omega)
\end{array}\right]\left[\begin{array}{c}
u(j \omega) \\
w_{1}(j \omega) \\
w_{2}(j \omega) \\
\vdots \\
w_{n}(j \omega)
\end{array}\right]
$$

In the above equation, $u(j \omega)$ represents control input and $w_{i}(j \omega)$ the $i^{\text {th }}$ exogenous disturbance. $y(j \omega)$ is the available feedback variable and $z_{i}(j \omega) s$ are the performance variables to be controlled but unavailable for feedback. The design objective is therefore to use only the feedback action $u(j \omega)=K(j \omega) y(j \omega)$ for simultaneous disturbance attenuation in both $y(j \omega)$ and $z_{i}(j \omega)$ for $i=1 \cdots n$.

To simplify the problem at discussion, it is further assumed that the exogenous disturbances $w_{i}(j \omega) s$ can be expressed as $w_{i}(j \omega)=C_{i} d(j \omega)$ for a frequency $\omega$ and $C_{i}$ is a complex number representing the gain and phase shift with respect to the same exogenous signal $d(j \omega)$. Then (1) can be rewritten as follows, dropping off the dependence on frequency for easy reference:

$$
\left[\begin{array}{c}
y \\
z_{1} \\
z_{2} \\
\vdots \\
z_{n}
\end{array}\right]=\left[\begin{array}{cccc}
G_{00} & G_{01} & \cdots & G_{0 n} \\
G_{10} & G_{11} & \cdots & G_{1 n} \\
G_{20} & G_{21} & \cdots & G_{2 n} \\
\vdots & \vdots & \ddots & \vdots \\
G_{n 0} & G_{n 1} & \cdots & G_{n n}
\end{array}\right]\left[\begin{array}{c}
u \\
C_{1} d \\
C_{2} d \\
\vdots \\
C_{n} d
\end{array}\right]
$$

Then with the only available feedback control $u=K y$ the performance response $y$ to exogenous signal $d$ is given:

$$
T_{y d}=\left(1-G_{00} K\right)^{-1} \sum_{k=1}^{n} G_{0 k} C_{k}
$$

The performance response of $z_{i}$ to $d$ is:

$$
T_{z_{i} d}=\left[1+\frac{G_{i 0} K\left(1-G_{00} K\right)^{-1} \sum_{k=1}^{n} G_{0 k} C_{k}}{\sum_{k=1}^{n} G_{i k} C_{k}}\right] \times \sum_{k=1}^{n} G_{i k} C_{k}
$$

The design objective can therefore be formulated as finding a controller $K$ such that $T_{y d}$ and $T_{z_{i} d}(i=1 \cdots n)$ 
are attenuated for a frequency $\omega_{0}$ or over a frequency band $\left[\omega_{1}, \omega_{N}\right]$. Clearly, to address this problem, the following questions must be resolved:

(1) The feasibility of simultaneous attenuation in both $y(j \omega)$ and $z_{i}(j \omega) s$ using only control $u=K y$;

(2) The level of attenuation in both $y(j \omega)$ and $z_{i}(j \omega) s$ wherever simultaneous attenuation is possible;

(3) Given a performance specification (e.g. attenuate $y(j \omega)$ by $6 \mathrm{~dB}$ while all $z_{i}(j \omega) s$ at least $3 \mathrm{~dB}$ etc), synthesize an optimal controller $K(j \omega)$ to fulfill the performance;

(4) Robustness issues associated with the optimal design.

The first question is of fundamental importance since answering existence questions is an important component of a good design methodology. However, an optimal controller should ultimately be produced. These problems are handled in the following sections.

\section{Discrete Frequency Controller Design}

To proceed, first consider the situation at a discrete frequency $\omega_{0}$. Thus to attenuate the disturbance in $y\left(j \omega_{0}\right)$, the feedback sensitivity $S \equiv\left(1-G_{00} K\right)^{-1}$ must be suppressed; similarly, disturbance in $z_{i}$ can be attenuated, provided that the disturbance response ratio for all $z_{i}\left(j \omega_{0}\right) s$ must be suppressed simultaneously. That is:

$\left|S\left(j \omega_{0}\right)\right|<1$

$\left|R_{i}\left(j \omega_{0}\right)\right|<1 \quad \forall i=1 \cdots n$

where:

$$
R_{i}=\left[1+\frac{G_{i 0} \sum_{k=1}^{n} G_{0 k} C_{k}}{G_{00} \sum_{k=1}^{n} G_{i k} C_{k}}(S-1)\right]
$$

Remark 1: The performance response of $y$ to $d$ in equation (3), and $z_{i}$ to $d$ in equation (4) can now be expressed as: $T_{y d}=S \sum_{k=1}^{n} G_{0 k} C_{k}$ and $T_{z_{i} d}=R_{i} \sum_{k=1}^{n} G_{i k} C_{k}$, hence $S$ and $R_{i}$ are the ratio of the closed-loop to the open-loop response with respect to the exogenous input $w_{i}$ (not $d$ ). Thus the original design objective can be reformulated as finding a controller $K$ such that the conditions (5) and (6) hold simultaneously for a frequency $\omega_{0}$ or over a frequency band $\left[\omega_{1}, \omega_{N}\right]$.

Remark 2: For discrete frequency control, either adaptive estimations (feedforward or feedback) or notch filters are used for suppressing the harmonic disturbance at $\omega_{0}$ in the field of noise and vibration control. These approaches are effective but will lose intuition into solution existence and limit of performance.

In the following, a pictorial method is developed to determine if a controller $K$ exists that satisfies conditions (5) and (6) simultaneously. The key to solve this problem is to notice that at a discrete frequency $\omega_{0}$, 
$S$ and all $R_{i}$ s are simply complex numbers. Therefore equation (6) can be mapped onto the complex $S$-plane in equation (5) through the Möbius transformation defined by (7). The result of the mapping is $n$ circles (call them $R$-circles) with circle-i having centre $\left(1-\operatorname{real}\left(G_{i}\right),-\operatorname{imag}\left(G_{i}\right)\right)$ and $\operatorname{radius}\left|G_{i}\right|$, where $G_{i}$ is defined as:

$$
G_{i}=\frac{G_{00} \sum_{k=1}^{n} G_{i k} C_{k}}{G_{i 0} \sum_{k=1}^{n} G_{0 k} C_{k}} \text { for } i=1 \cdots n
$$

This situation is illustrated in Fig. 1. It is noted that all the $R$-circles intersect unit $S$-circle at point $(1,0)$. At this point, $S=R_{i}=1 \forall i=1 \cdots n$. This implies that the performance responses of $y$ and $z_{i} s$ are simply their open loop responses and the controller $K=0$. For any other points, the levels of attenuation in $y$ and $z_{i}$ are the scaling with respect to the unit $S$-circle and $R_{i}$-circle respectively. For example, for a point with Cartesian coordinate $(\mathrm{a}, \mathrm{b})$, the attenuation or enhancement in $y$ and $z_{i}$ (closed loop performance) is $\sqrt{a^{2}+b^{2}}$ and $\frac{\sqrt{\left[a+\operatorname{real}\left(G_{i}\right)-1\right]^{2}+\left[b-\operatorname{imag}\left(G_{i}\right)\right]^{2}}}{\left|G_{i}\right|}$ depending on their values being smaller or greater than unity.

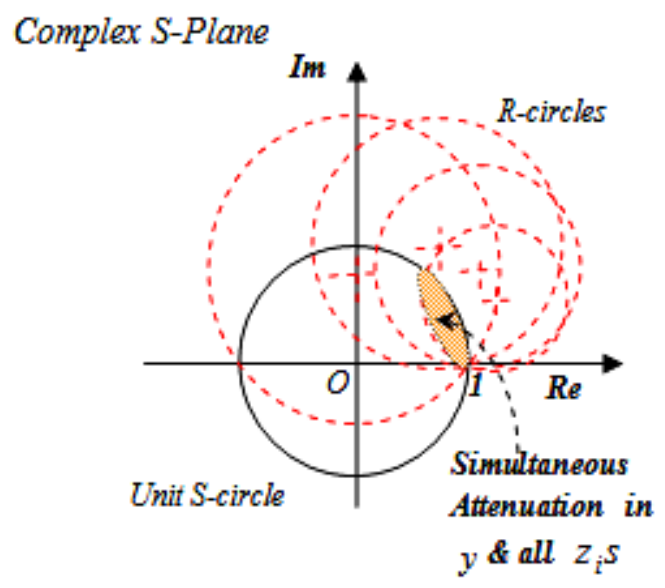

(a)

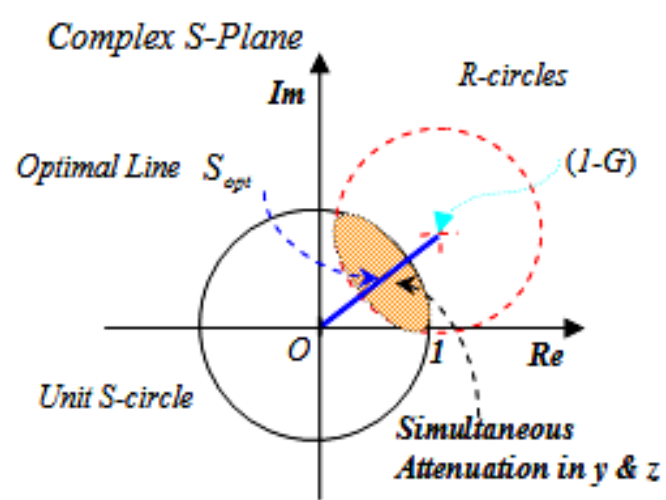

(b)

Fig. 1: Geometry of $S$ and $R$ revealing fundamental performance limitation at a discrete frequency

Figure 1(b) shows the situation for each $R_{i}$-circle. It is seen that an optimal line connecting the origins of the two circles can be naturally defined: at the origin of $S$-circle, disturbance at $y\left(j \omega_{0}\right)$ is annihilated while disturbance at $z_{i}\left(j \omega_{0}\right)$ is completely rejected at the origin of $R_{i}$-circle; moving the point on the optimal line results in a compromise in the level of attenuation in between $y\left(j \omega_{0}\right)$ and $z_{i}\left(j \omega_{0}\right)$. Thus the following important results follow from the inspection of Fig. 1:

Proposition 1 (Feasibility of Simultaneous Reduction): Simultaneous attenuation in $y(j \omega)$ and all $z_{i}(j \omega)$ $\forall i=1 \cdots n$ for a frequency $\omega_{0}$ is feasible if and only if there exists intersection among all the $R$-circles and unit S-circle. 
Proposition 2 (Level of Simultaneous Reduction): The level of reduction in $y(j \omega)$ and $z_{i}(j \omega)$ for a frequency $\omega_{0}$ is determined by the scaling with respect to the unit $S$-circle and $R_{i}$-circles, respectively.

Proposition 2 can be further delineated leading to a series of important results:

Result 1: A controller exists that provides infinite reduction in $z_{i}\left(j \omega_{0}\right)$ without any enhancement in $y\left(j \omega_{0}\right)$ if and only if the centre (1-G) locates inside the unit $S$-circle.

Result 2: A controller exists that provides infinite reduction in $y\left(j \omega_{0}\right)$ without any enhancement in $z_{i}\left(j \omega_{0}\right)$ if and only if the origin of the unit $S$-circle locates inside the mapped $R_{i}$-circle.

Result 3: A controller exists that will reduce one whenever the other is reduced if and only if $G=1$. In this case, the mapped $R_{i}$-circle coincides with the unit $S$-circle, and hence $y\left(j \omega_{0}\right)$ and $z_{i}\left(j \omega_{0}\right)$ are also attenuated by the same amount.

Now choosing a particular point on the complex $S$-plane implies designating a particular value for the sensitivity $S(j \omega)$ and this consequently means a particular design $K(j \omega)$ is obtained. This is the optimal controller for the discrete frequency control.

Proposition 3 (Optimal Controller Synthesis): The optimal controller $K(j \omega)$ achieving the performance specified by the optimal choice $S(j \omega)$ can be obtained as follows:

$$
K(j \omega)=\frac{S(j \omega)-1}{S(j \omega) G_{00}(j \omega)}
$$

Remark 3: Except at the special case $S(j \omega)=0$ meaning annihilation in $y(j \omega)$, which is impractical due to the requirement for infinite gain, the controller in (9) can be easily implemented at any discrete frequency using a recursive algorithm estimating the gain and phase shift of the harmonic signal.

The above results are of great practical importance since they suggest a method of resolving Higher Harmonics Control (HHC) that has been challenging in vibration and noise control problems. In the HHC problem, the disturbance is dominated by a discrete set of frequencies with higher harmonics often causing spillovers. To handle this problem, control is designed for each harmonic of the disturbance (superposition principle is applied). Thus the number of controllers required is increased with the increasing number of harmonics to be controlled. However, the results above suggest a distinctive solution to this problem.

Proposition 4 (HHC Problem): If there exists intersection among the R-circles of the higher harmonics to be controlled, then all the higher harmonics can be controlled by a single controller; if this intersection further intersects the unit $S$-circle, then this single controller will provide simultaneous reduction in all the performance variables.

Remark 4: This result is remarkable since it can be used to reduce the number of controllers for the HHC problem. This can substantially reduce the complexity and cost associated with the implementation of the control system. 


\section{Broad Band Control and Performance Improvement}

With the fundamental results for discrete frequency control, the problem of finding a controller $K$ such that the conditions (5) and (6) hold simultaneously over a frequency band $\left[\omega_{1}, \omega_{N}\right]$ can be resolved. But it is to be seen that this is a much involving problem since closed loop stability has to be concerned. It is also noted that the optimal choice $S(j \omega)$ is to be varied from one frequency to the other over the target frequency band. This results in an optimal trajectory on the complex S-plane. However a further constraint should be put on the design freedom $S(j \omega)$ so that closed loop stability is ensured. This can approached by defining $\Omega(j \omega)=S(j \omega)-1$, then it can be proved if $\Omega(j \omega)$ is a mapping of a stable function that also interpolates the unstable zeros of $G_{00}(s)$, then the resulting controller will internally stabilize the closed loop system for a stable but non-minimum phase $G_{00}(s)$. For a stable and minimum phase plant, the only constraint on the trajectory of $\Omega(j \omega)$ will be simply a mapping of a stable function. That is, if $\Omega(j \omega)$ is a mapping of a stable function, then the resulting compensator will internally stabilize the closed loop system for a stable and minimum phase $G_{00}(s)$.

However it is a well-known result that a stabilizing controller can itself be unstable. In practice, it will be desirable to have a stable and stabilizing controller, e.g. if the feedback loop opens due to sensor or actuator failure, an unstable controller can become problematic. The following main result solves this strong stabilization problem.

Proposition 5: If $G_{00}(s)$ is both stable and minimum phase and $\Omega_{o p t}(j \omega)$ is a mapping of a stable function and, in addition, $\operatorname{Re}\left(\Omega_{\text {opt }}\right)>-1$ when $\operatorname{Im}\left(\Omega_{\text {opt }}\right)=0$, then the resulting controller will not only internally stabilize the closed loop system and but also be itself stable.

Proof: It is first noted that the loop gain can be described by:

$$
L(j \omega)=K(j \omega) G_{00}(j \omega)=\frac{\Omega(j \omega)}{1+\Omega(j \omega)}
$$

The expression is equivalent to a closed loop system with loop gain $\Omega(j \omega)$ ), thus $L$ will be stable if $\Omega(j \omega)$ is also stable and its mapping of the Nyquist $D$-contour does not enclose the $(-1,0)$ point on the complex plane. Both of the stability conditions can simultaneously be met by ensuring that the $\Omega(j \omega)$ always crosses the real axis to the right of the $(-1,0)$ point. If now $G_{00}(s)$ is stable and minimum phase, then for a stable $L, K(s)$ will be stable and there will be no unstable pole-zero cancellations between $G_{00}(s)$ and $K(s)$. This completes the proof.

Remark 5: The assumption that $G_{00}(s)$ is both stable and minimum phase is not unduly restrictive since it is very likely that the sensor and actuator can be arranged to be collocated. As a consequence, the positive realness of $G_{00}(s)$ can be achieved. But it will be also reminded that the controller realization algorithm to be developed below can be used to account for a non-minimum phase $G_{00}(s)$. 
Now the optimal controller still needs to be constructed from the optimal choise $S(j \omega)$ over the frequency band $\left[\omega_{1}, \omega_{N}\right]$. From Proposition 5 , this is essentially a problem of finding a stable transfer function $\Omega(s)$ that interpolates the data points defined by $\Omega(j \omega)$ over $\left[\omega_{1}, \omega_{N}\right]$. This turns out to be a generalized Nevanlinna-Pick interpolation problem whose answer is provided by a modifed Pick condition [23]:

Modifed Pick Condition for Stable Interpolation: A stable transfer function $\Omega(s)$ that interpolates the optimal choice defined by $\Omega_{o p t}(j \omega)$ over $\left[\omega_{1}, \omega_{N}\right]$ exists if and only if the Pick matrix $P$ is positive definite:

$P=\left[\frac{1-\Omega_{k} \overline{\Omega_{l}} / M^{2}}{j\left(\omega_{k}-\omega_{l}\right)+2 a}\right]_{1 \leq k, l \leq N}$, where $\Omega_{i}$ is the optimal choice $\Omega_{\text {opt }}\left(j \omega_{i}\right)$ for frequency $\omega_{i} \forall i \in[1, N] ;$ $a$ and $M$ are positive real numbers defining the minimal degree of stability and maximum modulus of $\Omega(s)$ on the half plane $\mathfrak{R}(s) \geq-a$.

However, that $P$ fails to be positive definite would imply that the desired transfer function $\Omega(s)$ does not exist. The best appoximation to the optimal data points must be found. This can be achieved through a series of linear matrix inequalities (LMIs), with some of which defines the uncertainty bounded around each data points $\Omega_{o p t}\left(j \omega_{i}\right)$ for frequency $\omega_{i}$ over the frequency band $\left[\omega_{1}, \omega_{N}\right]$. By gradually relaxing the uncertainty bound, the best appoximation to the optimal data points will be eventually obtained. The desired stable transfer function $\Omega(s)$ that best interpolates the optimal choice is then obtained for either transfer function or statespace representation [24]. Manipulating Equation (9) leads to the optimal controller $K(s)=\frac{\Omega(s)}{[1+\Omega(s)] G_{00}(s)}$.

Remark 6: It is noted that the above optimal controller will be guranteed to provide best approximation to the performance specified in both $y(j \omega)$ and $z_{i}(j \omega) s$ over the frequency band $\left[\omega_{1}, \omega_{N}\right]$. Conventioanly, this problem is to be formulated as a mixed sensitivity problem such as $\left\|\begin{array}{c}W_{s}(s) S(j \omega) \\ W_{1}(s) R_{1}(j \omega) \\ \vdots \\ W_{n}(s) R_{n}(j \omega)\end{array}\right\|_{\infty} \leq \gamma$. While there exist rule-of-thumbs to choose the weighting functions to shape the sensitivities, it is still difficult to specify the performance or exploit performance limitations over any frequency band, nevertheless to tell if a performance specification in sensitivities is feasible or not.

Remark 7: It remains to see that the above procedure for optimal controller synthesis is a universal method to performance improvement to current control design, be it $H_{2} / H \infty$ or PID, over any desired frequency band $\left[\omega_{1}, \omega_{N}\right]$. This is achieved by utilizing the data points generated by current control, and the proposed method is then utilized to exploit the limit of performance for best achievable specification. The resulting controller will 
improve the performance over the frequency band $\left[\omega_{1}, \omega_{N}\right]$ while scarifying performance outside that frequency band (due to Bode's integral relationship). If the to-be-rejected disturbance is known to be in a specific frequency band, the proposed method will be beneficiary and certainly of significance to many practical systems.

\section{Disturbance Attenuation for a Rotor Blade Structure: Discrete Frequency Control}

In this section, a practical example is provided for discrete frequency control. Consider a rotor blade structure where disturbance enters through blades propagating along the shaft, the system is represented in (11) and the scenario is that only feedback control $u=K y$ is available to control both $y$ and $z$ at a frequency $\omega$. $u$ represents the force applied at the base of the rotor shaft, $y$ is the acceleration at the base of the shaft and $z$ is the acceleration on the rotor blade.

$\left[\begin{array}{l}y(\mathrm{j} \omega) \\ z(\mathrm{j} \omega)\end{array}\right]=\left[\begin{array}{l}G_{00}(\mathrm{j} \omega) G_{01}(\mathrm{j} \omega) \\ G_{10}(\mathrm{j} \omega) G_{11}(\mathrm{j} \omega)\end{array}\right]\left[\begin{array}{l}u(\mathrm{j} \omega) \\ d(\mathrm{j} \omega)\end{array}\right]$

Fig. 2 (a) shows the measured frequency response of the blade acceleration to excitation of the blade (the plot is hence of $\left|G_{11}(j \omega)\right|$ ). The first bending mode resonance in the region of $244 \mathrm{~Hz}$ clearly leads to a peak in the transmission along the shaft as shown in the measured base response $\left|G_{01}(j \omega)\right|$ in Fig. 2 (b).

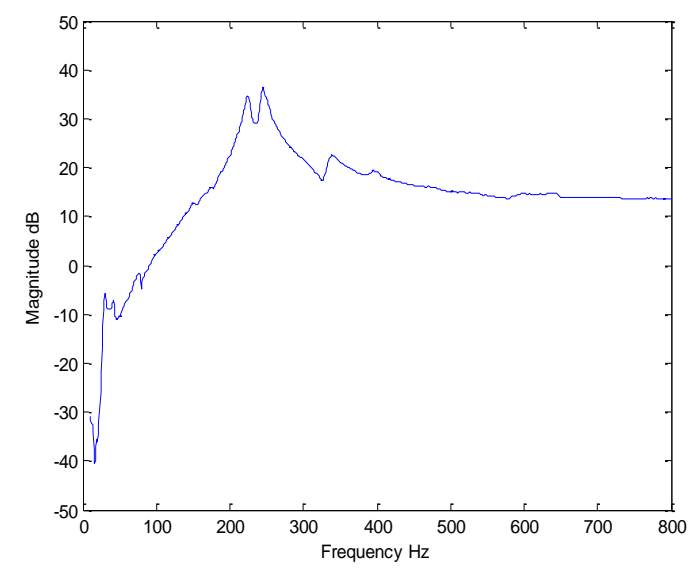

(a)

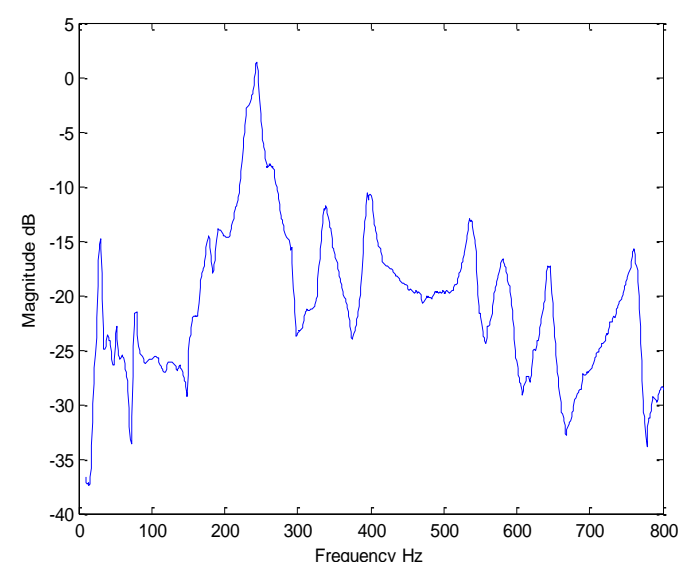

(b)

Fig. 2: (a) blade response to blade excitation $\left(\left|G_{11}(j \omega)\right|\right)$; $\quad$ (b) Base response to blade excitation $\left(\left|G_{01}(j \omega)\right|\right)$. Now map the R-circle onto the complex S-plane through the Möbius transformation defined by (7) at $\omega=244 \mathrm{~Hz}$. The resulting geometry of $R$ and $S$ is shown in Fig. 3. Also shown (dotted circles) are the mappings that represent a $6 \mathrm{~dB}$ attenuation boundary for $y$ and $z$, respectively. 


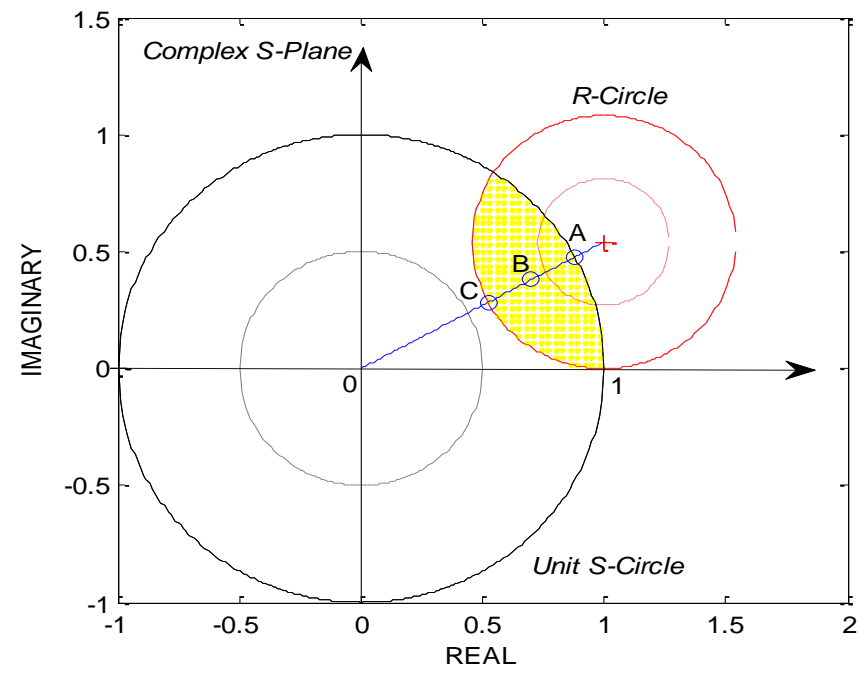

Fig. 3: Geometry of $S$ and $R$ for $\omega=244 \mathrm{~Hz}$.

Feasibility of Simultaneous Reduction: From Fig. 3, it is seen that there is a significant area of intersection between the unit $S$-circle and R-circle. And it follows from Proposition 1 that simultaneous attenuation in $y(j \omega)$ and $z(j \omega)$ for $\omega=244 \mathrm{~Hz}$ is feasible.

Level of Reduction and Optimal Control Design: Now choose 3 points labelled $A, B$ and $C$ in Fig. 3 and construct the corresponding controller respectively. Since point $A$ lies on the boundary of the unit S-circle and well within the $6 \mathrm{~dB}$ boundary of the R-circle, Proposition 2 shows that the resulting optimal controller will leave $y(j \omega)$ unchanged while reducing $z(j \omega)$ by a little over $11 \mathrm{~dB}$; conversely, the optimal controller with choosing point $C$ will reduce $y(j \omega)$ by a little under $6 \mathrm{~dB}$ without increasing $z(j \omega)$; finally point $B$ lies on the midpoint of intersection, and its location indicates the resulting optimal controller will reduce both $y(j \omega)$ and $z(j \omega)$ by around $3 \mathrm{~dB}$. The optimal controller for these three cases can be constructed from equation (9) of Proposition 3. Each of them can then be implemented using the recursive least squares algorithm for the condition of fixed amplitude $244 \mathrm{~Hz}$ sinusoidal disturbance forces. The simulation results are shown as acceleration time-histories in Fig. 4. The instant at which the controller is turned on in each case will be noted by the disturbance. It is seen clearly that the controller performs as predicted.
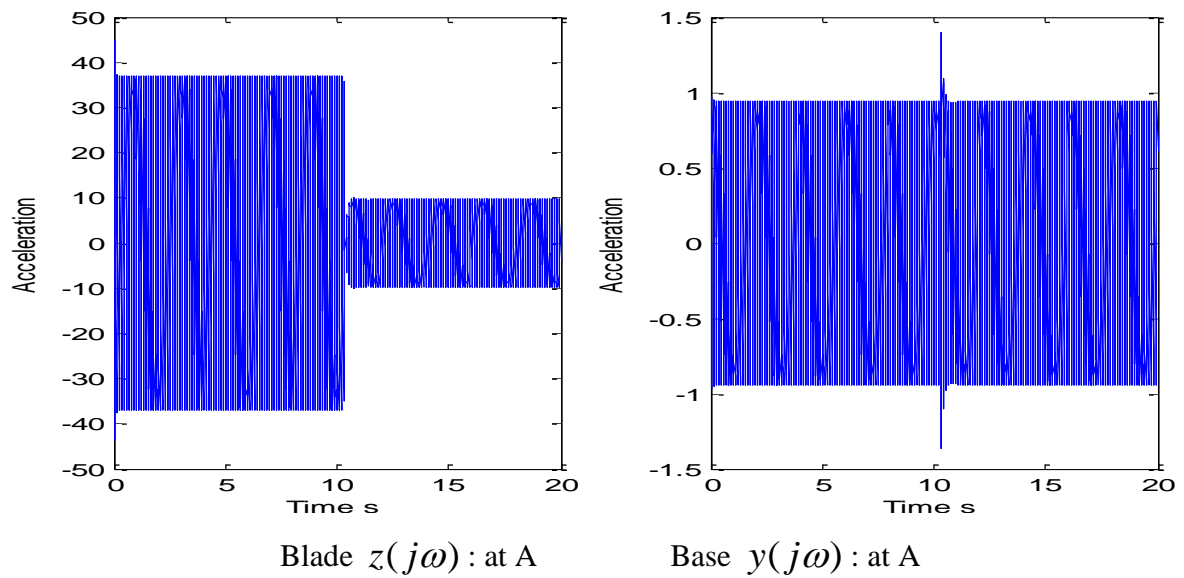

Blade $z(j \omega):$ at $\mathrm{A}$ 

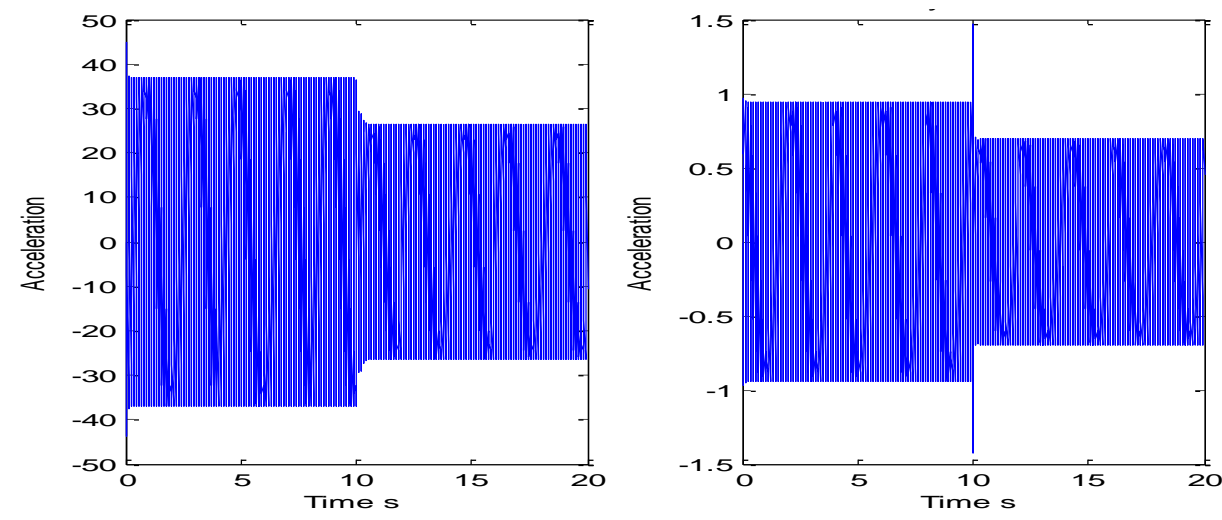

Blade $z(j \omega):$ at B
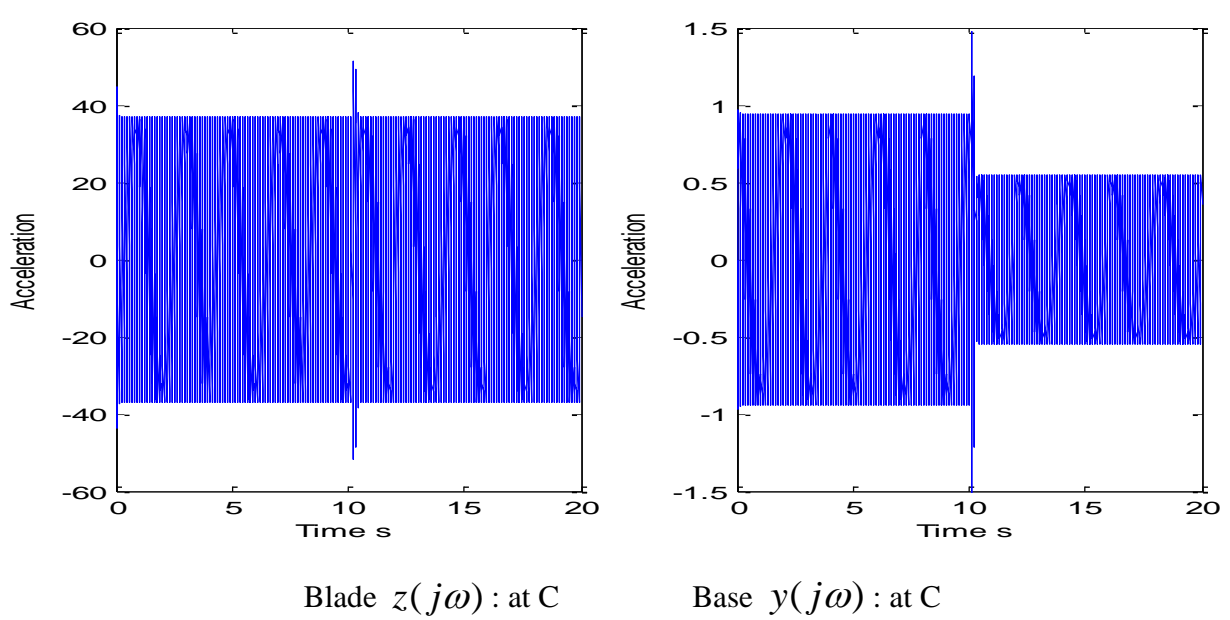

Fig. 4: Acceleration Time-Histories Each of the $244 \mathrm{~Hz}$ Controllers

Finally it will be worth pointing out that the design approach presented here is of great significance particularly when dealing with the practical difficulties associated with large scale interconnected systems. In the case of rotor blade structure there are numerous difficulties relating to the practical implementation of actuators and sensors into rotating frames. To resolve these problems, two distinctive solutions have been proposed: one is to confine attentions to analysis of individual elements [25], e.g. on shaft control [26] or on blades [27]; the other is to integrate smart materials directly into the blades acting as actuators, for example in references [28] and [29], and thus to treat the rotor blade structure as an integrated system. However, the former solution can cause serious problems since optimal control on the shaft can substantially enhance the vibration in blades and vice versa; the latter solution is very expensive and unproven in real working conditions. What has been shown above is that it is possible to control the whole structure using only shaft-based control actions, and thus demonstrate the feasibility of controlling a relatively complex system using a simple control strategy.

\section{Broad Band Control and Optimal Controller Performance Improvement}

Consider the broad band case for the region of $244 \mathrm{~Hz}$, e.g. over the frequency band [240, 250] Hz. Suppose now the following performance specification is given:

Performance Specification: Design a controller that will provide maximum attenuation in $z(j \omega)$, but without enhancement in $y(j \omega)$ over the frequency band $[240,250] \mathrm{Hz}$. 
Then the geometry of $S$ and $R$ can be plotted frequency-by-frequency as shown in Fig. 5, the above performance specification can be realized by the optimal choices that are indicated by the circles. It is seen that the required performance can be achieved if the modified Pick matrix is positive definite. While it is unfortunate that for the optimal data points, $P$ is not positive definite, henceforth a series of LMIs is formulated defining a small bound around the optimal choices. It turns out that only a slight modification to the above optimal choices will lead to a positive definiteness of $P$, implying that the achievable performance is very close to the performance specification, that is: the resulting controller will provide maximum attenuation in $z(j \omega)$ while not amplifying the disturbance in $y(j \omega)$ over the frequency band [240, 250] Hz. This is confirmed by the result in Fig. 6.

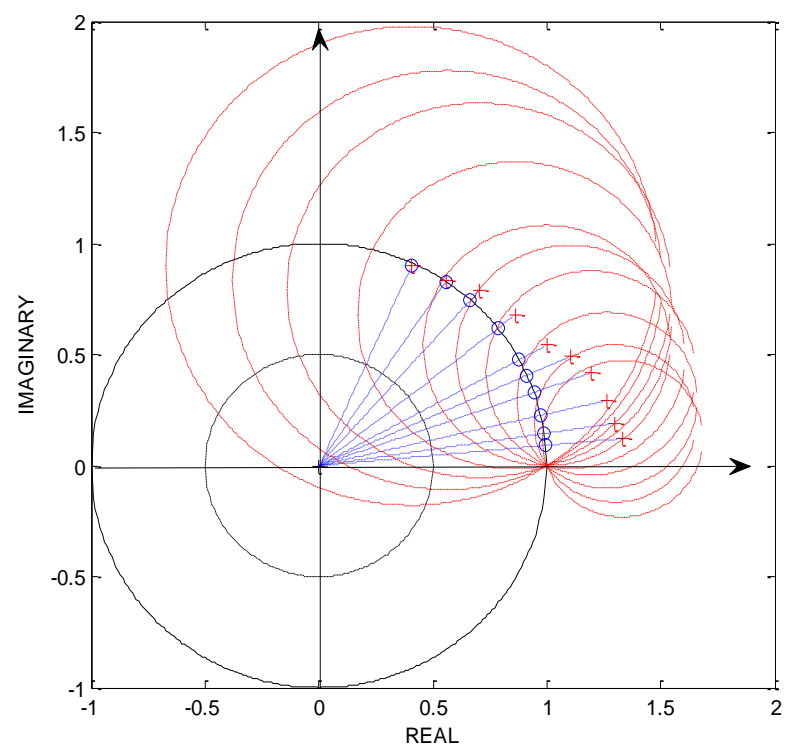

Fig. 5: Geometry of $S$ and $R$ over [240,250] Hz: the red slash-dot circles are $R$-circles; the blue dotted lines are optimal lines for each $S$ - $R$ geometry; while the circles are the optimal choices that are determined by the performance specification.

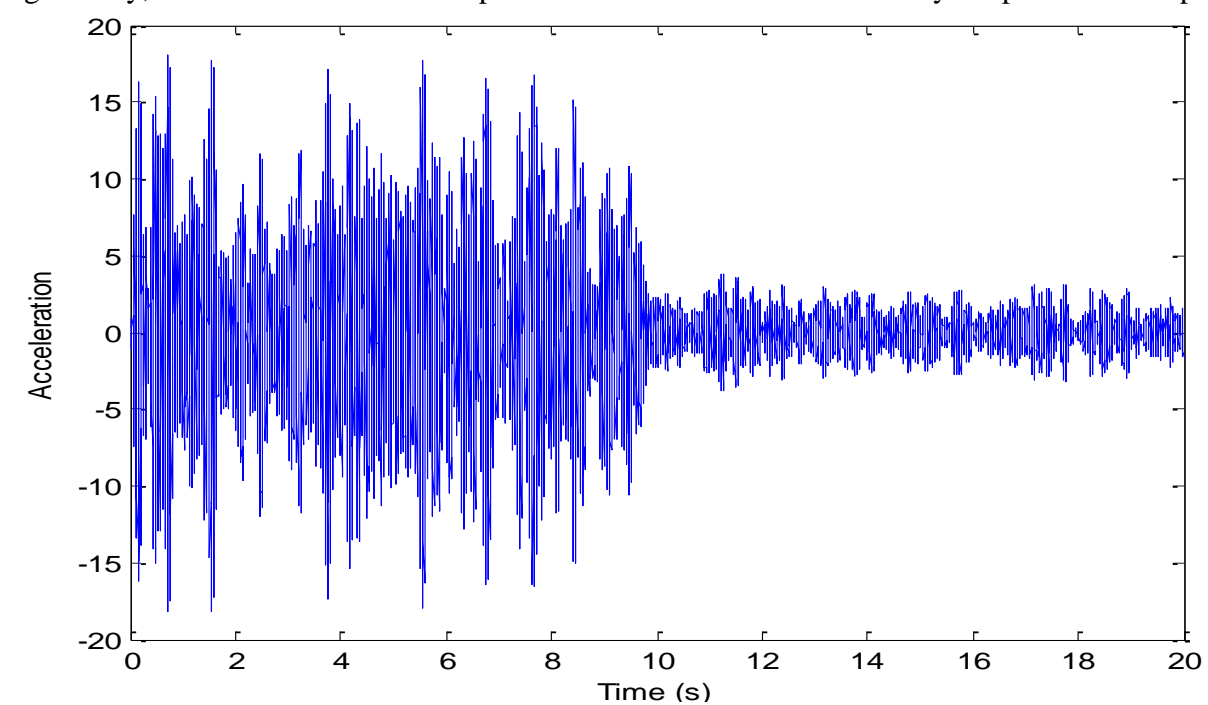

(a) 


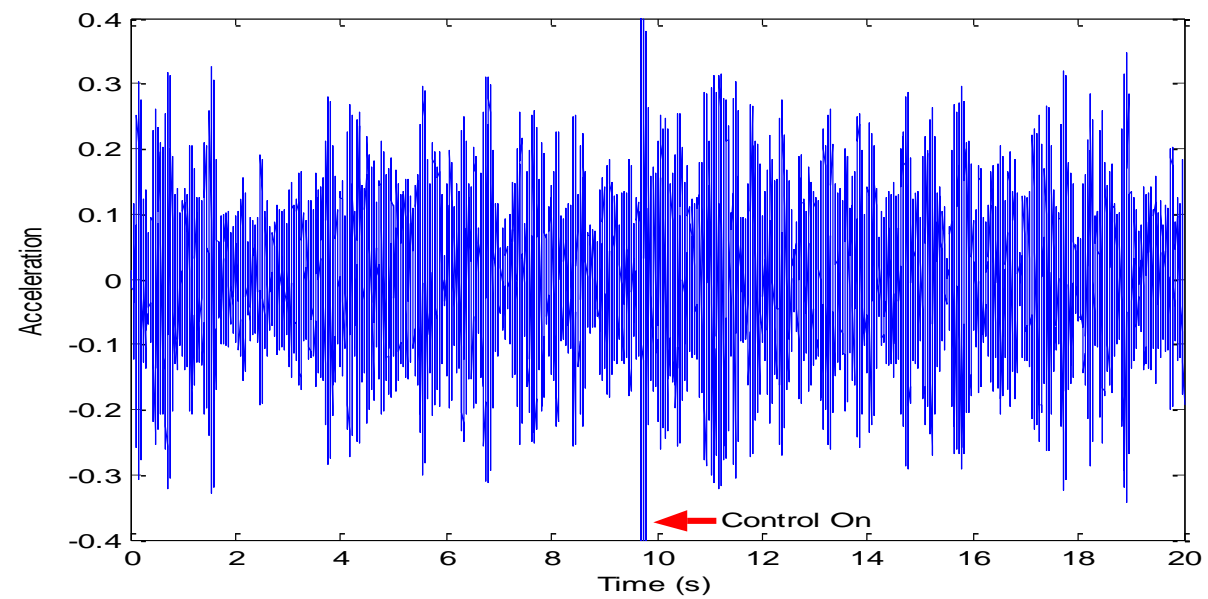

(b)

Fig. 6: On-line Performance of Resulting Controller (a) Significant Attenuation in $z(j \omega)$ (b) Disturbance is not enhanced in $y(j \omega)$

Now the more interesting thing is to demonstrate that the proposed method can be a universal method to performance improvement to current control design over any desired frequency band $\left[\omega_{1}, \omega_{N}\right]$. An $H \infty$ controller is to be constructed for the rotor blade example, resulting in a performance at the blades in Fig. 7.

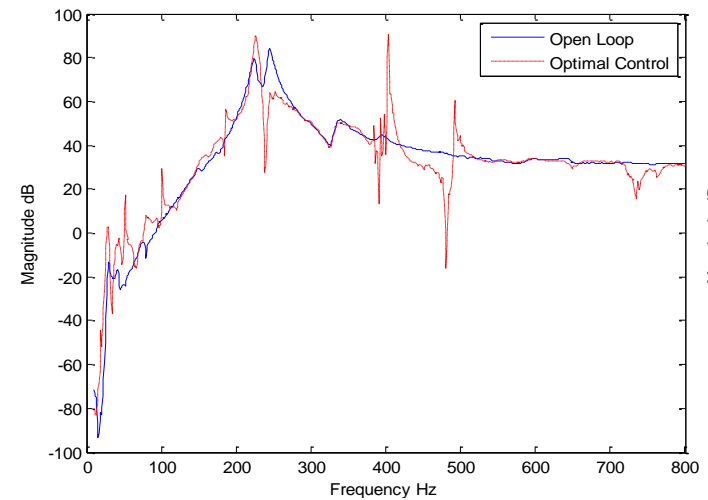

(a)

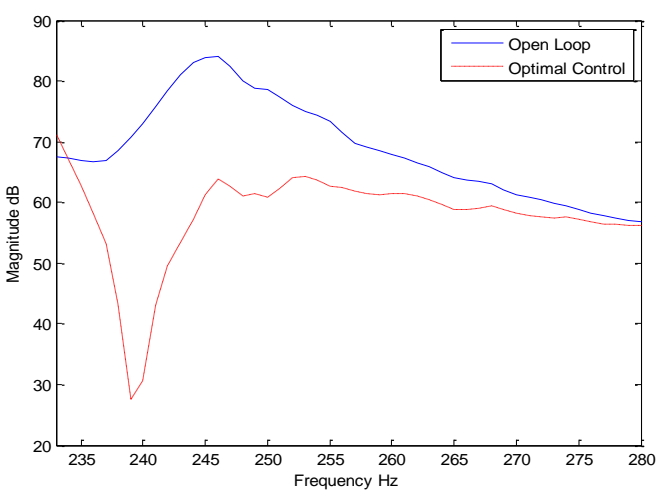

(b)

Fig. 7: (a) optimal controller performance for blade response to excitation; (b) magnified view over frequency band [240, 280] Hz. Significant vibration suppression is achieved over this frequency band.

Although the $H \infty$ controller performance can be further improved through, e.g. weighting functions, the problem of tailing performance over any pre-specified frequency band is still difficult to solve. As already commented, the proposed method can be used for such a purpose based on the $H \propto$ controller.

\section{Case I: In-band Performance Improvement}

From Fig. 7, it is seen that the peak around $244 \mathrm{~Hz}$ is "dragged down" by the Hळ controller, and thus disturbance is attenuated significantly over the first bending mode resonance. However it is also noticed that there is a second bending mode resonance at $225 \mathrm{~Hz}$, where unfortunately the resulting $\mathrm{H} \infty$ controller fails to attenuate the disturbance $(\mathrm{H} \infty$ control, by definition, always tries to minimize the frequency response function at the frequency with maximum magnitude.) Now the objective is to achieve attenuation over the frequency band covering both the two bending modes resonances. Application of the proposed methodology over, e.g. [200, $300] \mathrm{Hz}$, gives the result in Fig. 8 . 


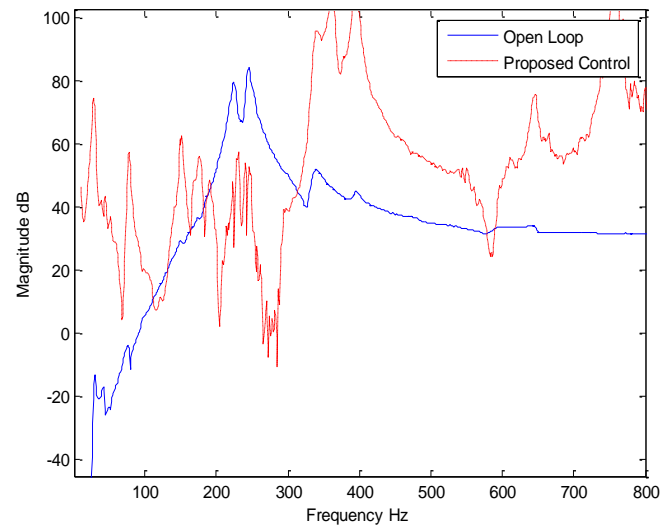

(a)

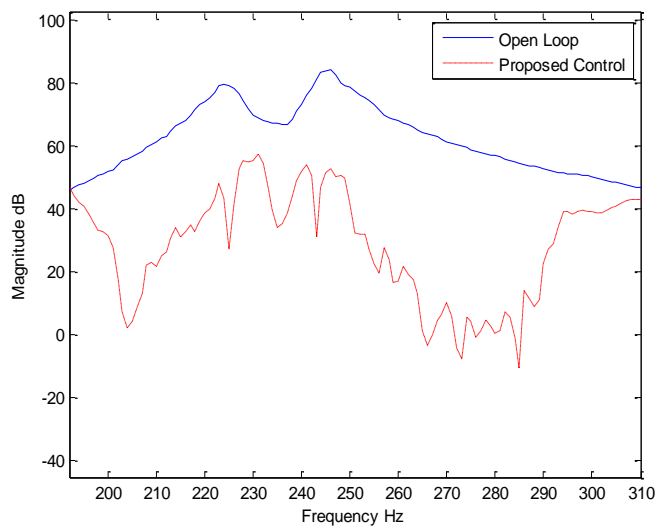

(b)

Fig. 8: (a) proposed controller performance for blade response to excitation; (b) magnified view over frequency band [180, 310] Hz. Vibration suppression is achieved over this frequency band covering both the first and the second bending modes.

It is seen that the proposed method improves the $\mathrm{H} \infty$ controller performance by sacrificing the performance outside, while significantly extending the effective frequency band from [240, 280] Hz to [180, 310]Hz. In the case where it is known that there is no disturbance force over the targeted frequency band, e.g. if there is no disturbance outside $[200,300] \mathrm{Hz}$ in the rotor blade system, then it is desirable to improve the Hळ controller performance over this frequency band without any concern upon the performance outside that frequency band. In fact, as the frequency spectrum of the disturbance entering the system can be estimated in practical engineering (or a band-pass filter can be utilized), it is meaningful to ignore the out-band performance deterioration while concentrating only on the desired frequency band, e.g. covering both the first and the second resonance modes, other than only one of them.

\section{Case II: Out-band Performance Improvement}

However, if the out-band performance is indeed a concern, then the proposed method can be utilized to re-shape the performance over required frequency band. In practice, it might be concerned either low frequency band or high frequency band. An application of the methodology over [100, 200]Hz provides improved performance over the $\mathrm{H} \infty$ control over this low frequency band as shown in Fig. 9 (a); while an application of the methodology over $[300,500] \mathrm{Hz}$ provides improved performance over the $\mathrm{H} \infty$ control over this high frequency band as shown in Fig. 9 (b). Thus the proposed method can indeed be utilized to re-shape control performance over a desired frequency band depending on practical interest.

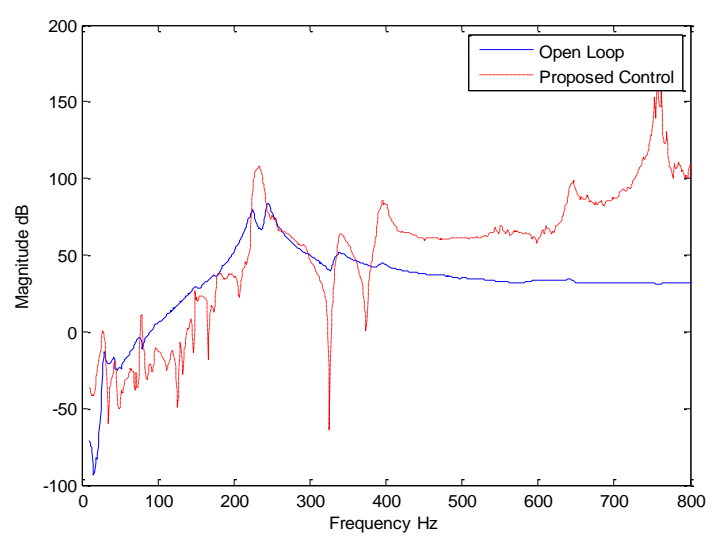

(a)

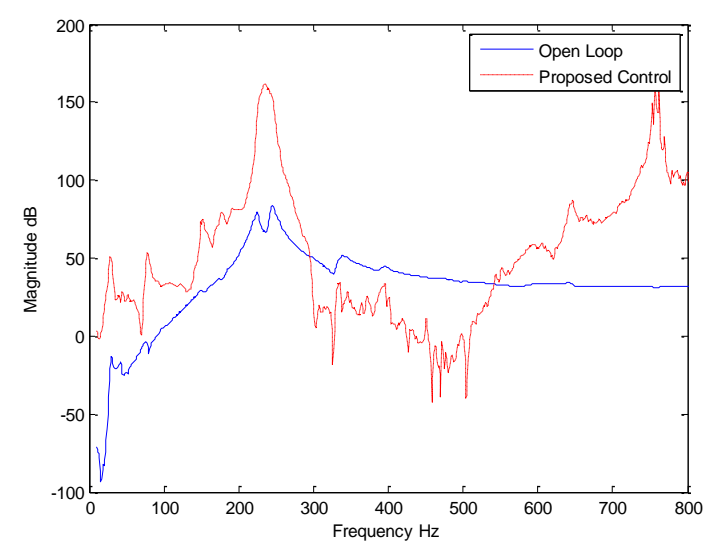

(b)

Fig. 9: Control performance improvement over (a) low frequency; (b) high frequency. 


\section{Conclusion}

Disturbance attenuation either at a discrete frequency or over a frequency band has been considered in this paper. A methodology has been proposed for addressing this problem. For many practical engineering systems, it is often feasible to estimate the spectrum of exogenous disturbance. There are cases where band-pass filters are used intentionally allowing disturbance with specific frequency band entering the system, e.g. radar or sonar detection \& anti-detection etc, then the problem of attenuating disturbance over (any) prescribed frequency band becomes important. The proposed method thus provides a framework to systematically tackling the underlying issues such as performance limitation, existence of controllers fulfilling prescribed performance, including the problem of improving the performance of an existing controller etc. Therefore the results presented in this paper provide new insights in the field of disturbance attenuation in linear systems.

Funding Acknowledgements: This work is supported by the Natural Science Foundation of Jiangsu Province (No. BK20140829); and the Fundamental Research Funds for the Central Universities (No. NS2016024).

\section{References}

[1] Francis, B.A., Helton, J.W., Zames, G. Optimal controllers for linear multivariable systems, IEEE Transactions on Automatic Control 1984, 29: 888-900.

[2] Francis, B.A. A Course in Control Theory, Springer-Verlag, New York, 1987.

[3] Doyle, J., Glover, K., Khargonekar, P., Francis, B.A. State-space solution to standard and control probelm, IEEE Transactions on Automatic Control 1989, 34(8): 831-842.

[4] Glover, K., Doyle, J. State space approach to optimal control, in Lecture Notes in Control and Information Sciences, Springer-Verlag, 1989, pp. 179-218.

[5] Zhou, K., Khargonekar, P., An algebraic Riccati equation approach to optimization, Systems and Control Letters 1998, 11: 85-91.

[6] Scherer, C., Gahinet, P., Chilali, M. Multiobjective output-feedback control via LMI optimization, IEEE Transactions on Automatic Control 1997, 42(7): 896-911.

[7] Arslan, G., Başar, T. Disturbance attenuating controller design for strict-feedback systems with structurally unknown dynamics, Automatica 2001, 37: 1175-1188.

[8] Jiang, Z.P. Nonlinear disturbance attenuation with global stability via output feedback, Proceedings of the 14th IFAC World Congress, Beijing, 1999.

[9] Cassandras, C.G., Lafortune, S. and Olsder, G.J. "Introduction to the modeling, control and optimization of discrete event systems," in Trends in Control, Isidori, A. (editor), Springer-Verlag London, 1995.

[10] Lou, Y., Hong, Y., "Target containment control of multi-agent systems with random switching interconnection topologies", Automatica 2012, 48: 879-885.

[11] Branicky, M.S., Borkar, V.S. and Mitter, S.K., "A unified framework for hybrid control: model and optimal control theory," IEEE Transactions on Automatic control 1998, 43(1): 31-45.

[12] Clarke, F., "Lyapunov functions and discontinuous stabilizing feedback”, Annual Reviews in Control 2011, 35: 13-33.

[13] Battistelli, G., Mari, D., Selvi, D., Tesi, A., Tesi, P. Adaptive disturbance attenuation via logic-based switching, Systems and Control Letters 2014, 73: 48-57.

[14] Antsaklis, P. and Baillieul, J. (editors), "Special Issue on Networked Control Systems," IEEE Transactions on Automatic Control 2004, vol. 49, no. 9. 
[15] Zhao, J., Hill, D.J., Liu, T., "Stability of dynamical networks with non-identical nodes: a multiple V-Lyapunov function method", Automatica 2011, 47: 2615-2625.

[16] Menon, P.P., Edwards, C., Postlethwaite, I. Optimal decentralised static output feedback stabilization of a network of dynamical systems, Systems \& Control Letters 2014, 64: 64-71.

[17] Landau, I.D., Alma, M., Constantinescu, A., Martinez, J.J., Noë, M. Adaptive regulation-rejection of unknown multiple narrow band disturbances (a review on algorithms and applications), Control Engineering Practice 2011, 19: 1168-1181.

[18] Montazeri, A., Poshtan, J. Adaptive IIR solution to active noise and vibration control systems, IEEE Transactions on Automatic Control 2010, 55(11): 2671-2676.

[19] Landau, I.D., Airimitoaie, T.B., Alma, M. IIR Youla-Kucera parameterized adaptive feedforward compensators for active vibration control with mechanical coupling, IEEE Transactions on Control Systems Technology 2013, 21(3): 765779.

[20] Zhang, S.Q., Schmidt, R., Müller P.C., Qin X.S. Disturbance rejection control for vibration suppression of smart beams and plates under a high frequency excitation, Journal of Sound and Vibration 2015, 353(29): 19-37.

[21] Kwakernaak, H. Mixed sensitivity design, in Proceedings of the 15th Triennial World Congress, Barcelona, Spain, 2002.

[22] Ortega, M.G., Rubio, F.R. Systematic design of weighting matrices for the Hœ mixed sensitivity problem, Journal of Process Control 2004, 14: 89-98.

[23] Ferreres, G., Puyou, G. Feasibility of $H \bowtie$ design specifications: an interpolation method, International Journal of Control 2005, 78(12): 927-936.

[24] Coelho, C., Silveira, L., Philips, J. Passive constrained rational approximation algorithm using Nevanlinna-Pick interpolation, Proceedings of the conference on Design, Automation and Test in Europe, 2002, pp.923-931.

[25] Chun, S.B. and Lee, C.W., "Vibration analysis of shaft-bladed disk system by using substructure synthesis and assumed modes method," Journal of Sound and Vibration 1996, 189(5), pp. 587-608.

[26] Bas, A., Gilheany, J. and Steimel, P., "Active vibration control of propeller shafts," Journal of Sound and Vibration 1998, 136 (3), pp. 361-372.

[27] Chen, Y., Wickramasinghe, V. and Zimcik, D., "Smart spring impedance control algorithm for helicopter blade harmonic vibration suppression," Journal of Vibration and Control 2005, 11, pp. 543-560.

[28] Chen, P.C. and Chopra, I., "Wind tunnel testing of a smart rotor with individual blade twist control," Journal of Intelligent Material Systems and Structures 1997, 8, pp. 414-425.

[29] Straub, F.K., Ngo, H.T., Anand, V. and Domzalski, D.B., "Development of a piezoelectric actuator for trailing edge flap control of full scale rotor blades," Smart Materials and Structures 2001, 10, pp. 25-34. 